\title{
Zusammenfassung und Abschluss
}

Die verschiedenen technischen Aspekte der Elektronik und Software im Fahrzeug, der Softwareentwicklung und -tests sowie der Prozessmodelle wurden aus unterschiedlichen Blickwinkeln betrachtet und in das Gesamtbild der Disziplin der Fahrzeuginformatik integriert. Das gerichtete Vorgehen und gelebte Prozesse der automobilen Praxis stehen mehr als die konkrete Technologie und Umsetzung als Schlüsselfaktoren in der Automobilindustrie im Vordergrund. Nur mit diesen Praktiken der Fahrzeuginformatik können die Herausforderungen an die Entwicklung der Produkte der Elektromobilität von morgen und damit der Mobilität der Zukunft gemeistert werden.

Bei der Anwendung der vermittelten Inhalte muss berücksichtigt werden, dass sich Erkenntnisse, Methoden und Vorgehensweisen genauso schnell weiterentwickeln können wie die zugrunde liegenden Technologien. Damit sollen die dargestellten Methoden einen Leitfaden und Vorschläge für die Entwicklung innovativer Produkte und im weitesten Sinne eine nachhaltige Mobilität geben. Sie erheben dabei im Gegensatz zur grundsätzlichen Fahrphysik und Elektronik keinen Anspruch auf technische Allgemeingültigkeit oder Vollständigkeit für alle Anwendungsfälle, da der Faktor Mensch einen wesentlichen Einfluss hat.

Die Methoden unterliegen damit auch der kontinuierlichen Änderung sowie Anpassung und leben vom Rückfluss der Erfahrungen der Anwender. Jeder Anwender und Entscheider muss für seinen Einflussbereich bewerten, welche der vorgeschlagenen Methoden anwendbar sind oder angepasst werden müssen. Das gilt vor allem für den kommenden Umbruch durch die Digitalisierung im Fahrzeug.

Der übergreifende Anspruch der Politik und Automobilindustrie nach ökologischer vertretbarer Mobilität muss auch volkswirtschaftlich nachhaltig sein. Das impliziert die Wirtschaftlichkeit technischer Konzepte, da sonst die Fahrzeuge entweder zu teuer werden oder der Wirtschaftsstandort nicht überlebensfähig ist. Die eingeführte Disziplin der Fahrzeuginformatik liefert dazu einen wesentlichen Beitrag. 